\title{
Curve severity and apical vertebral rotation and their association with curve flexibility in adolescent idiopathic scoliosis
}

\author{
S. P. Mohanty ${ }^{1} \cdot$ M. Pai Kanhangad ${ }^{1} \cdot$ A. Gullia ${ }^{1}$
}

Received: 22 March 2019 / Accepted: 11 April 2020 / Published online: 22 April 2020

(c) The Author(s) 2020

\begin{abstract}
Purpose To determine the association between coronal Cobb's angle and Nash-Moe index in patients with adolescent idiopathic scoliosis. We also attempted to determine whether apical vertebral derotation depended upon the curve flexibility. Overview of literature The three-dimensional nature of adolescent idiopathic scoliosis (AIS) is well established. Knowledge of all components of this complex deformity is essential to formulate effective treatment strategies. Though the importance of quantifying all the components of the deformity, in AIS, has been analysed in detail, very few studies have been done to ascertain the relationship between the coronal plane deformity and apical vertebral rotation.

Methods Digitalised standing and supine stretch anteroposterior (AP) radiographs of 158 patients with AIS were analysed. The standing and supine stretch AP radiographs were compared to calculate the percentage reduction of Cobb's angle to determine curve flexibility. The derotation of the apical vertebra on application of traction was also noted. The one-way repeated ANOVA was used to determine the association between Cobb's angle and Nash-Moe index. The independent sample $t$ test was used to determine whether a statistically significant difference was present, in the age of the patient, severity of the curve and percentage reduction of Cobb's angle between those curves that derotated and those that did not, when stretched. Results The one-way repeated ANOVA revealed an association between Cobb's angle and Nash-Moe index on the standing and supine AP stretch radiographs $(P<0.01)$. The Independent sample $t$-test showed a statistically significant difference in percentage reduction of Cobb's angle between those curves that derotated compared to those that did not, on stretch $(P<0.01)$.

Conclusions This study demonstrates that there is an association between apical vertebral rotation and the coronal plane deformity. It also demonstrates that flexible curves derotate to a greater extent compared to rigid curves, when stretched.
\end{abstract}

Keywords Adolescent idiopathic scoliosis $\cdot$ Apical vertebral rotation $\cdot$ Nash-Moe index $\cdot$ Cobb's angle

\section{Introduction}

Idiopathic scoliosis is the commonest type and accounts for nearly $80 \%$ of all structural scoliosis [1]. The three-dimensional nature of adolescent idiopathic scoliosis (AIS) is well established. Apart from the obvious lateral curvature, the spine is hypokyphotic at the apex and the vertebral bodies rotate towards the convexity of the curve [2]. Knowledge of all components of this complex deformity is essential to formulate effective treatment strategies. Early surgical

M. Pai Kanhangad

kmadhavapai@gmail.com; madhava.pai@manipal.edu

1 Division of Spine Surgery, Department of Orthopaedics, Kasturba Medical College, Manipal Academy of Higher Education, Manipal, Karnataka 576104, India principles primarily addressed the correction of the coronal plane deformity by distraction [3]. In recent times, there has been a growing consensus among surgeons, to address the three-dimensional nature of this deformity $[4,5]$.

The severity of the curve is routinely measured on an anteroposterior (AP) radiograph. The Cobb's angle is considered to be the gold standard in measuring the lateral curvature, as it closely corresponds to the magnitude of the deformity [6]. It is easy to perform, with a low intra- and interobserver variability [7]. Measurement of apical vertebral rotation (AVR) is an integral part of AIS evaluation, as it predicts risk of progression and is necessary for planning levels of instrumentation. Various methods have been described to determine AVR, most of which are based on the evaluation of the relative position of the posterior elements $[8,9]$. Cobb's method is not reliable as it does not correlate 
with vertebral rotation in degrees. The Perdriolle method is less reliable when the vertebral rotation is greater than $30^{\circ}$ [10]. While the computed tomography-based methods allow a more precise measurement of vertebral rotation, it requires a prohibitively high radiation dose [11]. Using predefined vertebral shape parameters like the angle from the pedicle to the spinous process and the distance from the pedicle to the vertebral body centre, Drerup developed a trigonometrical model to measure AVR [12, 13]. Even though the Nash-Moe index is an approximate measure of vertebral rotation, it is still one of the most popular methods used in clinical practice [14]. Recognition of the sagittal plane deformity has improved the understanding of AIS and has been integrated into newer classification systems [15].

Though the importance of quantifying all the components of the deformity, in AIS, has been analysed in detail, very few studies have been done to ascertain the relationship between the coronal plane deformity and AVR. This study was carried out to determine the association between coronal Cobb's angle and Nash-Moe index in patients with AIS. We also attempted to determine whether the reduction in AVR depended upon the flexibility of the curve.

\section{Materials and methods}

In a retrospective study, 354 consecutive patients presenting with scoliosis between the age of ten and eighteen years, from January 2012 to December 2017, were analysed. A clinical examination was done to detect neurological deficit, congenital anomalies and other causes of scoliosis. Standardised standing AP and lateral radiographs, supine lateral bending and stretch AP radiographs of the whole spine were obtained in all patients. They also underwent magnetic resonance imaging of the whole spine to rule out craniovertebral and spinal cord anomalies. One hundred and seventy-eight cases of congenital, neuromuscular, scoliosis secondary to tumours and infection were excluded. Twenty-seven patients with craniovertebral and cord anomalies were also excluded. Thus, 158 patients with AIS formed the basis of this study. Institutional ethical committee clearance was obtained prior to the commencement of the study.

\section{Radiological evaluation}

Digitalised radiographs were analysed by two independent observers (MPK and AG) in a blinded fashion using RadiANT software version 4.6.5. In case of a difference in the two readings, the decision of the senior observer was considered to be final. The upper end, lower end and apical vertebrae were identified on the standing AP radiographs. All curves were classified according to the Lenke's classification [15]. The severity of the curve was measured using the method described by Cobb et al. [6]. The Nash-Moe index was used to determine the rotation of the apical vertebra [8]. Similar method was used to determine the severity of the curve and AVR in the supine stretch film. The standing and supine stretch AP radiographs were compared to calculate the percentage reduction of Cobb's angle to determine curve flexibility. The derotation of the apical vertebra on application of traction was also recorded. In cases with more than one curve, these measurements were taken for all the structural curves. Thus, 196 curves in 158 patients were analysed. The Risser index was noted in all patients [16].

\section{Statistical analysis}

All statistical analyses were done using Statistical Package for Social Sciences for Windows version 18. The intraclass correlation and kappa coefficients were used to determine the intra- and interobserver variability for Cobb's angle and Nash-Moe index, respectively. Continuous variables like age, Cobb's angle and percentage reduction of Cobb's angle were expressed as mean \pm SD. Percentages were used to express categorical variables like Nash-Moe index. The one-way repeated ANOVA was used to determine the association between Cobb's angle and Nash-Moe index on standing and supine stretch AP radiographs. It was also used to compare the Cobb's angle in the thoracic, thoracolumbar and lumbar spine. Post hoc Tukey's test was done to determine whether a statistically significant difference was present between the various Nash-Moe grades. Spearman correlation coefficient was used to determine the relationship between age of the patient, severity of the curve and percentage reduction of Cobb's angle on stretch. The independent sample $t$-test was used to determine whether a statistically significant difference was present, in the age of the patient, severity of the curve and percentage reduction of Cobb's angle between those curves that derotated and those that did not, on stretch. The Chi squared test was used to compare the frequency of the various Nash-Moe grades in the thoracic, thoracolumbar and lumbar spine. It was also used to compare the frequency of Risser 0, 1, 2 incurves that derotated and those that did not, when stretched. Receiver operating characteristic (ROC) of the curve was performed to assess the area under the curve (AUC) of age, standing Cobb's angle and percentage reduction of Cobb's angle in relation to apical vertebral derotation. A $P$ value less than 0.05 was considered to be statistically significant.

\section{Results}

The inter- and intraobserver agreeability for Cobb's angle was 0.93 and 0.98 , and for Nash-Moe index, it was 0.61 and 0.86 , respectively. The mean age of patients was 
$14.5 \pm 2.1$ years. The male-to-female ratio was $1: 6.75$. The most common curve pattern was Lenke type 1B. The ratio of right-sided to left-sided curves was 2.37:1. The most common apical vertebrae were T9, L1 and L2 in the thoracic, thoracolumbar and lumbar spines, respectively. The mean Cobb's angle was $51.26 \pm 17.43^{\circ}$ and $28.28 \pm 14.97^{\circ}$ on the standing and supine stretch AP radiographs, respectively. Supine stretch AP radiographs showed a 46.2 \pm 16.1 percent reduction of Cobb's angle. The details of curve severity, flexibility and AVR are shown in Table 1.

The one-way repeated ANOVA revealed an association between Cobb's angle and Nash-Moe index on the standing and supine stretch AP radiographs $(P<0.01)$. Post hoc Tukey's test showed a statistically significant difference in Cobb's angle between the three Nash-Moe grades, on the standing as well as supine stretch AP radiographs $(P<0.01)$. A higher Nash-Moe index was associated with a higher Cobb's angle. Bivariate analysis revealed a weak negative correlation between Cobb's angle on standing AP and percentage reduction of Cobb's angle on supine stretch AP radiographs (Spearman's rho $=-0.243$, $P=0.01$ ); however, there was a poor correlation between age and percentage reduction of Cobb's angle (Spearman's rho $=-0.131, P=0.07)$. In the supine stretch AP radiographs, the reduction in Cobb's angle was accompanied with a reduction in Nash-Moe index in a total of 77 curves, whereas in 119 curves it showed no change. The Independent sample $t$-test showed a statistically significant difference in percentage reduction of Cobb's angle between those curves that derotated compared to those that did not, when stretched $(P<0.01)$. However, no statistically significant difference in age and Cobb's angle was noted between the two groups [Table 2]. The frequency of Risser $0,1,2$ was higher in curves that derotated compared to curves that did not $(46.75 \%$ vs $27.73 \%)(P=0.018)$.
Table 2 Factors affecting curve derotation

\begin{tabular}{llll}
\hline & $\begin{array}{l}\text { Curves that derotated } \\
\text { by one Nash-Moe grade } \\
(n=77)\end{array}$ & $\begin{array}{l}\text { Curves that did } \\
\text { not derotate } \\
(n=119)\end{array}$ & $P$ value \\
\hline Age & $14.23 \pm 1.68$ & $14.68 \pm 2.28$ & $0.142^{*}$ \\
$\begin{array}{l}\text { Cobb's } \\
\text { angle } \\
\text { (standing }\end{array}$ & $51.98 \pm 17.64$ & $50.8 \pm 17.36$ & $0.644^{*}$ \\
$\begin{array}{l}\text { AP) } \\
\text { Percentage } \\
\text { reduction } \\
\text { of Cobb's } \\
\text { angle }\end{array}$ & $50.66 \pm 13.69$ & & \\
\hline
\end{tabular}

*Independent sample $t$-test

The ROC analysis revealed AUC of 0.44, 0.51 and 0.63 for age, standing Cobb's angle and the percentage reduction of Cobb's angle in relation to apical vertebral derotation [Fig. 1]. The critical value of 48 percentage reduction of Cobb's angle had a sensitivity of $61 \%$, a specificity of $59 \%$, a positive predictive value of $70 \%$ and a negative predictive value of $49 \%$, for derotation of the apical vertebra by one Nash-Moe grade, when stretched.

\section{Discussion}

The three-dimensional nature of scoliosis was well understood prior to the invention of radiography [2]. With the advent of radiographic evaluation of scoliosis, greater emphasis was assigned to the coronal plane deformity. In 1966, Scoliosis research society adopted the use of Cobb's angle for quantitative assessment of severity of the curve due to its reliability. The current study showed high inter- and intraobserver agreement for Cobb's angle, similar to that of previous studies $[7,11]$. This study

Table 1 Curve severity, flexibility and apical vertebral rotation

\begin{tabular}{|c|c|c|c|c|}
\hline & Thoracic $(n=113)$ & Thoracolumbar $(n=38)$ & Lumbar $(n=45)$ & $P$ value \\
\hline Cobb’s angle (mean \pm SD) & $55.41 \pm 17.16$ & $45.87 \pm 17.27$ & $45.39 \pm 15.56$ & $<0.01 *$ \\
\hline Traction Cobb's angle (mean \pm SD) & $32.5 \pm 15.65$ & $20.94 \pm 10.39$ & $23.85 \pm 12.91$ & $<0.01 *$ \\
\hline Percentage reduction of Cobb's angle & $42.42 \pm 15.69$ & $54.28 \pm 15.18$ & $48.92 \pm 15.11$ & $<0.01 *$ \\
\hline \multicolumn{5}{|l|}{ Standing radiographs } \\
\hline Nash-Moe grade 1 & 42 & 8 & 12 & \multirow[t]{3}{*}{$0.477 * *$} \\
\hline Nash-Moe grade 2 & 62 & 23 & 30 & \\
\hline Nash-Moe grade 3 & 9 & 7 & 3 & \\
\hline \multicolumn{5}{|l|}{ Supine stretch radiographs } \\
\hline Nash-Moe grade 1 & 78 & 22 & 27 & \multirow[t]{3}{*}{$0.119^{*}$} \\
\hline Nash-Moe grade 2 & 30 & 15 & 17 & \\
\hline Nash-Moe grade 3 & 5 & 1 & 1 & \\
\hline
\end{tabular}

*One-way repeated ANOVA, **Chi squared test 


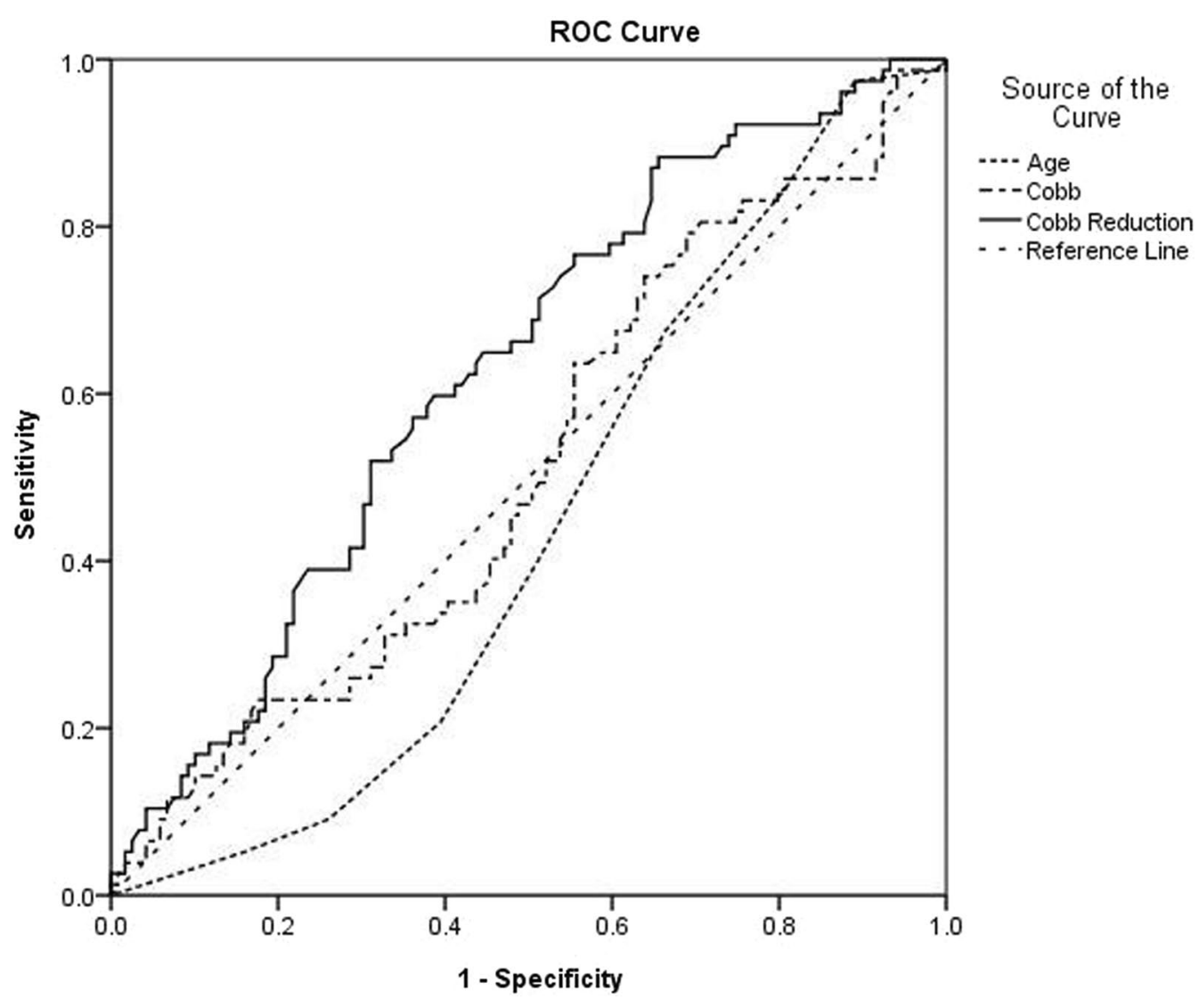

Fig. 1 Receiver operating characteristic curves for age, standing Cobb's angle and the percentage reduction of Cobb's angle in relation to apical vertebral derotation

showed significantly less severe curves in the thoracolumbar and lumbar spines. This is similar to the findings of other authors [17]. While various techniques have been described to quantify the rotational deformity, the Nash-Moe index is still widely used in clinical practice. This study demonstrated that there was no significant difference in the frequencies of various Nash-Moe grades in the thoracic, thoracolumbar and lumbar spines. This is consistent with the findings of Stoke et al.[18].

The origin and progression of the deformity in AIS are not well understood. Various theories have been proposed regarding the development and progression of the rotational deformity in scoliosis. Lovett and Arkin observed that coupling of lateral bending and axial rotation is a kinematic property of the normal spine $[19,20]$. While studies have shown that there is some nonlinear relationship between the elemental components of the deformity, its exact nature is not clear. Our study showed that there was a statistically significant difference in the Cobb's angle between the various Nash-Moe grades, and the higher Nash-Moe grades had higher Cobb angles. This is similar to the findings of Sullivan et al. and Morrison et al., who found a correlation between Cobb's angle and AVR [21, 22].
This study demonstrated that more severe curves were less flexible, while age did not significantly affect curve flexibility. Mathematical models have shown that ribs increase the stiffness of the thoracic spine in scoliosis [23]. This probably explains why the reduction of the coronal Cobb, upon stretch, was significantly lower in the thoracic compared to the thoracolumbar and lumbar spines, in this study. Kadoury et al. studied the effects of various types of instrumentation on vertebral rotation. They observed that though some derotation was seen with Harrington distraction instrumentation, better correction was possible with newer techniques [17]. In the current study, 77 curves derotated by one Nash-Moe grade, whereas 119 curves showed no change upon stretching. Curves that derotated had a significantly higher flexibility compared to curves that did not. However, no significant difference in age and Cobb's angle was noted between the two groups. ROC analysis showed that curves with coronal Cobb that reduced by $48 \%$ in supine stretch films had a tendency to derotate by one Nash-Moe grade with a sensitivity of $61 \%$ and a specificity of $59 \%$.

Early surgical principles primarily addressed the lateral curvature of the spine. Though satisfactory results were obtained with Harrington's distraction instrumentation, the 
current study showed distraction alone did not completely address all components of the deformity [17]. Cotrel and Dubousset shifted the premise of curve correction from distraction to derotation of the curve about the axial plane [4]. However, various studies have shown that this concept is not entirely correct. Thus, newer correction manoeuvres that employ a combination of derotation and translational forces may achieve better three-dimensional deformity correction and restoration of global spinal alignment [24]. Timing of surgery is also important in achieving optimum correction of the deformity [25]. Therefore, surgical correction should be carried out before the curve progresses in severity and becomes rigid.

The strengths of this study include its large sample size and detailed statistical analyses, some of which have not been previously done. While the Nash-Moe index is commonly used in clinical practice, it is only a rough estimate of AVR with a $25^{\circ}$ interval between each grade. Being a complex three-dimensional deformity accurate measurement of vertebral rotation is difficult. Another drawback of the current study is the inability to standardise the stretch force applied while obtaining supine AP radiographs. While CT scan is considered to be superior to other methods, it measures rotation in the supine position. Atmaca et al. used the method described by Drerup to determine vertebral rotation from preoperative standing radiographs. This technique may be of use in preoperative evaluation of scoliosis and post-operative curve decompensation below the lowest instrumented vertebra [26]. Future studies comparing the different methods can be done to determine the best measure of AVR, in order to analyse the exact relationship between AVR and coronal plane deformity.

This study shows that there is an association between AVR and the coronal plane deformity. It also demonstrates that flexible curves derotate to a greater extent compared to rigid curves, when stretched.

Acknowledgments Open access funding provided by Manipal Academy of Higher Education, Manipal.

\section{Compliance with ethical standards}

Conflict of interest The authors declare that they have no conflict of interest.

Ethical approval All procedures performed in studies involving human participants were in accordance with the ethical standards of the institutional and/or national research committee and with the 1964 Helsinki Declaration and its later amendments or comparable ethical standards. Institutional ethical clearance was obtained prior to the start of the study.

Human and animal rights This article does not contain any studies with animals performed by any of the authors.
Informed consent Informed consent was obtained from all individual participants included in the study.

Open Access This article is licensed under a Creative Commons Attribution 4.0 International License, which permits use, sharing, adaptation, distribution and reproduction in any medium or format, as long as you give appropriate credit to the original author(s) and the source, provide a link to the Creative Commons licence, and indicate if changes were made. The images or other third party material in this article are included in the article's Creative Commons licence, unless indicated otherwise in a credit line to the material. If material is not included in the article's Creative Commons licence and your intended use is not permitted by statutory regulation or exceeds the permitted use, you will need to obtain permission directly from the copyright holder. To view a copy of this licence, visit http://creativecommons.org/licenses/by/4.0/.

\section{References}

1. Willner S, Uden A (1983) A prospective prevalence study of scoliosis in southern Sweden. Acta Orthop Scand 53:233-237

2. Stokes IA, Bigalow LC, Moreland MS (1987) Three-dimensional spinal curvature in idiopathic scoliosis. J Orthop Res 5(1):102-113

3. Schultz AB, Hirsch C (1973) Mechanical analysis of Harrington rod correction of idiopathic scoliosis. JBJS 55(5):983-992

4. Dubousset J, Cotrel Y (1991) Application technique of CotrelDubousset instrumentation for scoliosis deformities. Clin Orthop Relat Res 264:103-110

5. Lee SM, Suk SI, Chung ER (2004) Direct vertebral rotation: a new technique of three-dimensional deformity correction with segmental pedicle screw fixation in adolescent idiopathic scoliosis. Spine 29(3):343-349

6. Cobb JR (1948) Outline for the study of scoliosis. Instr Course Lect AAOS 5:261-275

7. Morrissy RT, Goldsmith GS, Hall EC et al (1990) Measurement of the Cobb angle on radiographs of patients who have scoliosis. Evaluation of intrinsic error. J Bone Joint Surg 72(3):320-327

8. Nash CL, Moe JH (1969) A study of vertebral rotation. J Bone Joint Surg Am 51(2):223-229

9. Perdriolle R, Vidal J (1985) Thoracic idiopathic scoliosis curve evolution and prognosis. Spine 10(9):785-791

10. Richards BS (1992) Measurement error in assessment of vertebral rotation using the Perdriolle torsionmeter. Spine 17(5):513-517

11. Illés T, Somoskeöy S (2013) Comparison of scoliosis measurements based on three-dimensional vertebra vectors and conventional two-dimensional measurements: advantages in evaluation of prognosis and surgical results. Eur Spine J 22(6):1255-1263

12. Drerup B (1984) Principles of measurement of vertebral rotation from frontal projections of the pedicles. J Biomech 17(12):923-935

13. Drerup B (1985) Improvements in measuring vertebral rotation from the projections of the pedicles. J Biomech 18(5):369-378

14. Cerny P, Marik I, Pallova I (2014) The radiographic method for evaluation of axial vertebral rotation-presentation of the new method. Scoliosis 9(1):11

15. Lenke LG, Betz RR, Harms J, Bridwell KH, Clements DH, Lowe TG, Blanke K (2001) Adolescent idiopathic scoliosis: a new classification to determine extent of spinal arthrodesis. JBJS 83(8):1169-1181 
16. Biondi J, Weiner DS, Bethem D (1985) Correlation of Risser sign and bone age determination in adolescent idiopathic scoliosis. J Pediatr Orthop 5(6):697-701

17. Kadoury S, Cheriet F, Beauséjour M, Stokes IA, Parent S, Labelle H (2009) A three-dimensional retrospective analysis of the evolution of spinal instrumentation for the correction of adolescent idiopathic scoliosis. Eur Spine J 18(1):23-37

18. Stokes IA, Bigalow LC, Moreland MS (1986) Measurement of axial rotation of vertebrae in scoliosis. Spine 11(3):213-218

19. Lovett RW (1905) The mechanism of the normal spine and its relation to scoliosis. Boston Med Surg J 153(13):349-358

20. Arkin AM (1950) The mechanism of rotation in combination with lateral deviation in the normal spine. JBJS 32(1):180-188

21. Sullivan TB, Bastrom T, Reighard F, Jeffords M, Newton PO (2017) A novel method for estimating three-dimensional apical vertebral rotation using two-dimensional coronal cobb angle and thoracic kyphosis. Spine Deform 5(4):244-249

22. Morrison DG, Chan A, Hill D, Parent EC, Lou EH (2015) Correlation between Cobb angle, spinous process angle (SPA) and apical vertebrae rotation (AVR) on posteroanterior radiographs in adolescent idiopathic scoliosis (AIS). Eur Spine J 24(2):306-312
23. Schultz AB, Galante JO (1970) A mathematical model for the study of the mechanics of the human vertebral column. J Biomech 3(4):405-416

24. Vallespir GP, Flores JB, Trigueros IS, Sierra EH, Fernández PD, Olaverri JC, Alonso MG, Galea RR, Francisco AP, de Paz BR, Carbonell PG (2008) Vertebral coplanar alignment: a standardized technique for three dimensional correction in scoliosis surgery: technical description and preliminary results in Lenke type 1 curves. Spine 33(14):1588-1597

25. Mohanty SP, Kumar MN, Kishore BN, Babu SS (2001) The timing of spinal fusion in adolescent idiopathic scoliosis. J Orthop Surg 9(2):7-14

26. Atmaca H, Inanmaz ME, Bal E, Caliskan I, Kose KC (2014) Axial plane analysis of Lenke 1A adolescent idiopathic scoliosis as an aid to identify curve characteristics. Spine J 14(10):2425-2433

Publisher's Note Springer Nature remains neutral with regard to jurisdictional claims in published maps and institutional affiliations. 